\title{
EXPERT PANEL DISCUSSION
}

James M. Rippe, MD, Robert Boone, MBA, MHA, Amy Mechley, MD, Michael Parkinson, MD, MPH, Robert Porter, JD, MBA, and Dexter Shurney, MD, MBA, MPH

\section{American College of Lifestyle Medicine Expert Panel Discussion: Lifestyle Medicine Integrating Evidence Into Practice 2015*}

Editor's Note: The following Expert Panel discussion was recorded on November 1, 2015, in Nashville, Tennessee, as the opening session for the annual meeting of the American College of Lifestyle Medicine.

The following individuals participated:

- James M. Rippe, MD, is Founder and Director, Rippe Lifestyle Institute and Professor of Biomedical Sciences at the University of Central Florida. He serves as Editor in Chief, American Journal of Lifestyle Medicine.

- Robert Boone, MBA, MHA, is President and CEO of the Medical Fitness Association (MFA). As a pioneer in the Wellness and Fitness Industry, he brings a wealth of experience, including over 25 years as a hospital executive in operations to add to his extensive knowledge on creating and profitably operating medically integrated fitness centers.
- Amy Mechley, MD, is a board-certified family medicine physician and Medical Director of The Wellness Division at The Christ Hospital Health Network in Cincinnati, Ohio.
- Robert Porter, JD, MBA, joined Healthways early in 2013 after a long and distinguished career in health system leadership, predominantly with SSM Health Care. In September

\section{"The challenge for lifestyle medicine \\ is how do we distinguish it as something that is truly 'signal' and not just "more noise'?"}

- Michael Parkinson, MD, MPH, is the Senior Medical Director overseeing employer health and productivity strategies for UPMC Health Plan and WorkPartners. He formerly was Executive Vice President, Chief Health and Medical Officer of Lumenos, a national pioneer of consumer-directed health plans acquired by Wellpoint. of 2014, Bob assumed the role of Managing Director of the Ornish Program.

- Dexter Shurney, MD, MBA, MPH, is the Chief Medical Director/Executive Director for Global Benefits, Health and Wellness for Cummins, Inc. He has an extensive background in health care management and policy.

*This article is based on an Expert Panel Discussion delivered at the American College of Lifestyle Medicine 2015 Annual Meeting, November 2015.

DOI: 10.1177/1559827616638296. From Rippe Lifestyle Institute, Shrewsbury, Massachusetts (JMR); Medical Fitness Association, Pinehurst, North Carolina (RB); The Christ Hospital Health Network, Cincinnati, Ohio (AM); UPMC Health Plan and WorkPartners, Pittsburgh, Pennsylvania (MP); Ornish Reversal Program, Franklin, Tennessee (RP); and Cummins, Inc, Columbus, Indiana, USA (DS). Address correspondence to: James M. Rippe, MD, Rippe Lifestyle Institute, 21 North Quinsigamond Avenue, Shrewsbury, MA 01545; e-mail: bgrady@rippelifestyle.com.

For reprints and permissions queries, please visit SAGE's Web site at http://www.sagepub.com/journalsPermissions.nav. 
Dr Rippe: I am delighted to, once again, serve as the moderator for the Expert Panel at the Annual Meeting of the American College of Lifestyle Medicine (ACLM). I consider it a proud achievement that my team and I at Rippe Lifestyle Institute actually named the field "lifestyle medicine" with the publication of the first textbook of Lifestyle Medicine in 1996. The second edition of this textbook came out in 2013 (Lifestyle Medicine, 2nd ed. Boca Raton, FL: CRC Press; 2013). ${ }^{1}$ I should mention that I also edit the major intensive care textbook in America-Irwin and Rippe's Intensive Care Medicine, ${ }^{2}$ which outsells my Lifestyle Medicine textbook 20:1. Before I die, I hope that those numbers are reversed!

It is deeply gratifying for me to be here and see the wonderful growth of ACLM. I applaud the direction that the organization is taking to adopt the application of evidenced-based medicine in the area of lifestyle medicine. To deeply embed lifestyle medicine into the fabric of American health care will require not only evidence but creativity. Let me give you an example of what I regard as a creative application of lifestyle medicine evidence and principles.

The SPRINT Trial was a major cardiovascular intervention study supported by the National Institutes of Health. The trial enrolled 9300 adults who had increased risk of heart disease or kidney disease who were randomized to have their systolic blood pressure lowered either to $140 \mathrm{~mm} \mathrm{Hg}$ or to 120 $\mathrm{mm} \mathrm{Hg}$. The trial was stopped prematurely because individuals who got their blood pressure down to $120 \mathrm{~mm}$ $\mathrm{Hg}$ lowered their risk of heart disease and stroke by $33 \%$ and mortality from those diseases by $25 \%$.

One negative aspect of this trial was that to get blood pressure down to 120 $\mathrm{mm} \mathrm{Hg}$ required on average 3 medicines. I don't believe the average patient who sees us in our clinics would be willing to take 3 medicines to lower blood pressure. The reason I am telling you this is because if we could get people in our country to simply take 1 medicine and combine that with not smoking cigarettes, regular physical activity, and weight loss if necessary, I am quite confident that we could get most patients' blood pressure down to 120 $\mathrm{mm} \mathrm{Hg}$. This would be a wonderful example of lifestyle medicine at work!

Since more than $35 \%$ of individuals in our country have high blood pressure and it remains the leading cause of outpatient visits, this would lend considerable prominence to the role of lifestyle medicine. And of course, the recommendations that I have made here are also incorporated into national guidelines for managing high blood pressure promulgated by both JNC (Joint National Committee) VII and JNC VIII.

We will now ask the panelists to respond to 2 broad questions. First question: Describe what transformation from the current disease care system to a health care system looks like in the context of lifestyle medicine.

Dr Parkinson: Several perspectives shape my personal view on this topic. In addition to my role at UPMC, I also work with employers and health care organizations nationally to accelerate innovative change. I also am honored to work with DOD (Department of Defense) and through the IOM (Institute of Medicine), the VA Health System. As such, I am able to spend considerable time involved in policy in Washington, was President of the American College of Preventive Medicine during the formation of what is now known as "Obama Care" so I witnessed many of the inner workings of the legislative process that created the ACA (Affordable Care Act).

Let me offer an analogy. In the military and in neurology, there is something called "signal to noise ratio." The challenge for lifestyle medicine is how do we distinguish it as something that is truly "signal" and not just "more noise?" Some of the issues that I think are noise include ACOs (Accountable Care Organizations), bundled payments, reference pricing, care coordination fees, and short-term enhanced payments from CMS (Centers for Medicare and Medicaid
Services) to primary care. While all good, these near-term tactics largely represent noise because they do not address the root cause of our health, care, and cost problem. They all theoretically support the cause of lifestyle medicine and they are "good noise," but still noise, not signal.

So what does represent true signal? How about rethinking of what we call a "cancer?" Oftentimes lesions that are regarded as "early cancer" or "cancer in situ" really represent low-grade inflammation. In fact, a National Cancer Institute-commissioned panel came to the conclusion that our use of the word "cancer" should be seriously reviewed particularly for early stage disease. How about prostate cancer? It more often may represent reversible inflammation in the gland rather than true cancer. And Ornish has shown that "early prostate cancer" can be reversed with lifestyle interventions. That's the real signal. That's an impactful and transformative medical signal that places lifestyle medicine into the mainstream of medical diagnosis and treatment.

Sometimes we whine and complain that nobody pays for lifestyle medicine. I thought when I went to medical school and focused on family medicine I was already doing what lifestyle medicine was all about-namely, utilizing lifestyle as a first treatment option. I always told my patients to lose weight. I always told them to stop smoking and eat better. But they didn't. The problem was I had the wrong information and ineffective training and support to help them improve behaviors. I got so frustrated that I trained in preventive medicine because $80 \%$ of disease I saw was preventable-yet I was having little impact in traditional primary care. Bottom line: lifestyle medicine needs to get beyond the current noise, to leverage the noise in order to express the signal impact of addressing the root cause of disease. We have to get much more sophisticated in our both strategy and tactics to do so however. We have to be more laser focused on how we use changes in health care to promote lifestyle medicine as core to the solution 
rather than simply screaming from the mountain top. That never works. If lifestyle medicine is successful, we won't have to talk about lifestyle medicine anymore. It is the way we live. It is the way we practice medicine and it fulfills the promise that I initially pursued in family medicine. Today, more than then, there is better science and knowledge of what works to prevent, treat, and reverse disease. What's needed is more sophisticated approaches to leverage lifestyle medicine as core to so-called health care transformation.

Dr Mechley: A conference like this is tremendously supportive since it facilitates like-minded people to come together and synergize to create something better. Change starts in settings like this. The evolution of lifestyle medicine is what you are seeing in front of you. We are doing it and feeling it. I want to address two core tenets that I believe are critical for changing from a disease care system to a health care system. A change in payment and a change in culture. We currently have two generations of physicians and patients who are used to a fee for service system. The simplest thing would be to continue to do what we are doing. Unfortunately, that has gotten us to the broken place where we are now. We overtreat and overtest. We are still treating disease in ways that do not improve health and spending an enormous amount of money in doing so.

So the first tenet is a change in payment. I have been blessed to be chosen as a director to the Ohio area Comprehensive Primary Care Initiative (CPCI), which is a Center for Medicare and Medicaid Innovation (CMMI) project. These programs are funded to discover new models of health care that lower costs and improve outcomes. That's where the ACO Pioneer project came from. We know that the ACOs have not been as successful as we hoped they would be. Some of us saw that outcome early because we knew the drivers weren't changing. The ACOs were implemented in a varied way, most not involving the patient as a driver for change. It became a risk exchange in a capitated environment as opposed to getting to the root cause of improving healthcare outcomes

So we are in year 3 of our 4-year initiative. There are 7 areas across the country that are involved in this program. The difference that CPCI has, which I believed would make it successful, is that there was payment upfront-there was capital to invest in a new enhanced model of care. The payers said "we want you to transform primary care. We understand primary care is central in leading this and we are going to pay you for it. We are actually going to pay you upfront to hire a team and make infrastructure change." This part of the payment system is called a PMPM (per member per month payment) and is based on the empanelled patients. In the Cincinnati, Ohio and Northern Kentucky area, there are 9 commercial payers with CMS that were involved with this so it was very transformative-commercial payers as plus Medicare. In the second year of the program we were able to decrease Medicare spending by $1.5 \%$. We didn't just slow the curve down we actually bent it down. Unfortunately, because of the methodology, we did not gain any shared savings in our area but we were able to decrease the spending.

This is an important experiment to understand what we need to do to create change. The CPCI encouraged teambased care. This is where I see lifestyle medicine succeeding. It is the only current traditional model that I see in a fee for service system that lifestyle medicine has a chance of gaining the foothold. Because there is a PMPM upfront, and possible shared savings in the back, it is essential to decrease the total cost of care. I truly believe we need to do that through team based care. In CPCI, the PMPM continued to decline and if you look at the evidence, it is going to take about a \$5 PMPM to really do advanced primary care. To truly get to transformative lifestyle medicine, I believe we will need to be looking at $\$ 8$, which is supported by some recent literature.

The second core tenet is to change the culture, to empower healthy behaviors. It is easier now to access and to eat processed food. Seventy percent of all the food we eat in America is processed. One thing I always tell my patients is eat real food, actual food. Not food substitutes are, simply eat real food and move more.

A basic tenet of lifestyle medicine is making the right choice, the easy choice. It needs to be cool to be healthy. It needs to be the right thing to do. It needs to be a sense of national pride that we are actually healthy. We can change some of the drivers and the economics, but we understand that this is our culture right now and that we need to support each other in living a healthier lifestyle. Right now it is really cool to sit on the couch, play video games, and have so many conveniences that many things are done for you. We need to get up and get moving!

I have been named to Governor Kasich's State of Ohio Primary Care Redesign Team. At the state level, we are trying to duplicate some of the CPCI model and have received $\$ 75$ million from the federal government to do so. As we do this work and continue CPCI, I am more concerned that incremental change is not going to be effective. We need to blow this up. We need disruptive change. There is a model that will be talked about during the conference called "DPC" (Direct Primary Care). I think this is the model that will truly get us out of the stranglehold of fee for service medicine for primary care. It will break the bonds that we are restrained in right now with primary care and it will cause an integrated approach where lifestyle medicine can thrive. You're going to hear some experts talk about the DPC. I am a huge fan of this model knowing what I do now as I am immersed in payment reform with the insurance companies and the government on both the national and state level. Just keep an eye on DPC. I think that's where we are going to create true population health change.

Mr Porter: You will hear a lot of consistency across the various panelists. A number of issues have already been 
touched on. I believe we are at an inflection point. Clearly the current system is unsustainable. We are going to make 1 of 2 choices-either to really transform the system to one that can reduce the amount of avoidable demand in health care systems to make it affordable, inclusive, and high quality or we are going to cut the payments to the existing system, which will inevitably fall harder on some segments of the population than others. It is going to be incumbent on people who know where the waste is, where the opportunity is for us to work at making the health care system more sustainable and higher quality. I believe it is the people in this room that are going to lead that movement.

When I think about all of this in the context of lifestyle medicine, I want to emphasize several things. First, you have to start with a complete understanding of the nature of well-being and a holistic understanding of the determinants of health and well-being so we don't just think within the contours of the health care system itself for medicine. We think more broadly about social determinants of health, communities, and all of the factors that ultimately go into, not only the physical health of the person, but their emotional and spiritual health as well.

Second, we have to be able to really think about the notion of culture more broadly than just the culture of medicine or the culture of health care. We have to become leaders on the movement that reaches more broadly into our communities, as well as employers and into schools. We need to be vocal advocates for change so that the good behaviors that start in the office of a physician or primary care medical home can be carried on and supported more broadly in the community. That notion of culture really is going to be driven strongly by those who are paying the cost of health care. The employers are going to become much more active by trying to encourage and support the notion that the normative behavior is health behavior not the outlier.
Clearly, we have to rework how our funds flow to be able to encourage investment in the right things and we have to change our time horizon for these expenditures. We are looking in terms of a time frame within which return for investment in healthy behavior and changes in lifestyle can be rewarded but we have to be articulate in terms of return on investment. We need to recognize the people who are paying the bills need to see the business case. If we are going to be effective advocates for lifestyle medicine we have to speak in a language that reflects not only advocacy for what we want to spend money on, but how it generates a return on investment for those for those who are doing the financing.

Obviously, there is a radical redesign and delivery model and that radical redesign is one of the hardest things because we are talking about fundamentally changing the nature of how the health care system works. It has been through multiple generations of working the way it is. How are we going to begin to create comfort with people making that kind of radical change? Redefinition of primary care, not as a doctor or an office, but as a relationship with a patient that actively engages and equips them to be able to optimize their health and well-being, supported by a team of people rather than having the individual physician who waits for a complaint and treats it—really activating a team of people who are actively engaged with that person on an ongoing basis. We need to rethink the whole notion of centers for well-being and opportunities to get access to services before the person gets sick to mitigate risky behaviors. We need to rethink access to minor episodic care so that you get the right care, right place, right time, right provider. There is a whole overhaul that has to take place in the way in which we deliver care. That is tough because the financing and delivery system doesn't really reward this approach at this point in time. It is not going to be like we flip a switch and suddenly move from one system to another and do it effectively.
We need that commitment to evidencebased medicine, including recognition of the growing body of evidence that lifestyle changes are effective tools in the treatment, as well as prevention, of disease. We need to be rigorous in the science that supports this work that we are doing because there is a great deal of skepticism that it will work. That people will do it even if it does work. We have to be knowledgeable about the science of behavior change as well as the science of effective lifestyles. What are the conditions that make it more likely than not that an individual will make and sustain a change that will ultimately lead them to better health and well-being?

Finally, we need to use the powerful digital tools that are at our disposal to be able to both have better tools for decision making-more real-time availability of information, more patient motivation, and the kind of things that really can help us reduce and get rid of the variation that is avoidable in order to create a more active and engaged individual.

Dr Shurney: I am excited to be here. I am happy to see the remarkable group of people at this conference who are starting to understand and to grasp the importance of lifestyle medicine. This is an exciting time for lifestyle medicine but it is also an exciting time for physicians. Those of you that are practicing lifestyle medicine see how your patients respond to these techniques is just amazing. I have been in practice for a long time and I have never seen anything like the power of lifestyle medicine in terms of our patients. The satisfaction when someone is able to reverse their disease is incredibly rewarding. I have never seen anything like it. It is why we wanted to go medical school-to learn how to heal and make people feel better. Unfortunately, for most of my career, like many of you, I have just been handing out "Band-Aids." The patient continues to cut themselves and you give them another Band-Aid. But with lifestyle medicine, you stop the cutting, so there is less need for Band-Aids. Again, this is a really exciting time and I think we 
need to grab this opportunity and run with it. People are looking for real solutions and this is real. There is nothing fake or made up. This is not a fad. This is real.

What does the transformation look like? I can tell you based on what I do right now, as a physician working within a manufacturing environment, people are always talking about Six Sigma. It helps us work smarter and becomes the foundation of everything that we do. If there is a problem or there is an issue, you seek to address the problem at its root cause. We spend a lot of time training black belts and master black belts, but when it comes to health care we really haven't done that.

One of the things that excites me about my job is that we are now looking at how to take a Six Sigma approach to figuring out health care. Not just from a medical waste perspective, but broader. When they look at chronic disease from a Six Sigma, root cause approach, where does it take you? What is the root cause when it comes to most of chronic disease conditions? Most chronic diseases are preventable and are a matter of poor lifestyle. While we spend a lot of money, time, and effort trying to negotiate rates or trying to bundle payments with carriers and providers, we're missing the bigger opportunity of not paying for things that wouldn't happen if we could get to the root cause and do a better job on the lifestyle front.

So the answer to me relates to getting to the root cause and using the mitigating the root cause not only for prevention, but also as the treatment of disease, and in some cases to reverse disease. That is the opportunity. That's transformative. The transformation also looks not having to have a separate diabetes program, a separate heart program, a separate hypertension program-it's all one program. It's lifestyle medicine.

I am encouraged that as a society we'll achieve our goals because now I understand that lifestyle medicine is now beginning to be taught in medical schools, or at least being strongly considered. That is something that is long overdue. This is an exciting time because I believe we now have a critical mass of people that really get this, and that really understand it. We are going to run with it and because of the higher level of outcomes only possible through these means, we are going to make history!

Mr Boone: I, too, am really excited to be here. It has been a particular pleasure this year getting to know some of the team at the American College of Lifestyle Medicine. It is a great privilege for me to introduce the Medical Fitness Association at this conference. I think the collaboration between our organizations can pay great dividends for all of us.

When it comes to taking a look at the transformation of health care, you have heard 4 very distinguished individuals offer approaches to this. I believe we are all in sync about where transformation is going. I am going to put my hospital administrator hat on, however, for just a minute. I want to talk about the business case for transformation and what it might look like as we go down the road. The first issue, as all of you know, is we don't have a health care system in this country-we have a sick care system. We treat symptoms. We don't treat causes. And that is what excites me about this group. ACLM and its members are looking to treat the root causes of chronic disease.

Hospitals practice Six Sigma all the time but they often do it in the wrong way in my opinion. Many solutions are just surface fixes instead of taking a deeper dive. I think we are going to see medicine moving forward using a team concept and we will be providing more individualized medicine. We will seek more preventive solutions and look for ways to reverse disease instead of treating symptoms. How do we go about producing some accountability in health care? How do we transition away from the system says "you can go do anything you want to and when you get sick we are going to take care of you regardless?" We need to start putting some personal responsibility back on patients. Fully
" $80 \%$ of what goes wrong with us is because of what we put in our mouths or how we choose to live our lives." So why aren't we accountable for those decisions we make including some of the financial burden for our decisions. People who choose to live unhealthy lifestyles should bear some of that burden. There have been some failed efforts in the past, but I think you will see renewed efforts in this area. Many businesses are already implementing carrots and sticks for their employee populations by providing incentives for improving lifestyle choices and putting in place penalties for those who choose not to change. Initiatives like that are common. A former employer of mine, a hospital, has just put in place a requirement to have your BMI (body mass index) measured in order to keep the lowest level premium. Next year you will have to meet your recommended BMI plus $20 \%$, and the following year if you don't meet BMI there is going to be a penalty levied on your health care premium. So businesses are recognizing that the system we have now is not sustainable. As others have said, the only model I see out there that has the potential for being sustainable is to truly embrace prevention-primary, secondary, tertiary prevention, and lifestyle medicine is the approach.

Putting on my medical fitness hat now, what is this medical fitness thing and how does it fit in here? I view us in the medical fitness area really as a team member for physicians and other health care workers. Doctors have been saying all along to patients, "you need to exercise. You need to eat right," and so on. But guess what? You say that to a patient and often nothing happens. How many of your patients actually go out and exercise just because you say you should go exercise? Very few. That's where we in the medical fitness area come in.

As we move forward in working together, we have to look at what the data say. What evidence do we have to show that these lifestyle medicine programs work? How do we create outcomes that are meaningful to both 
you, as practitioners, so that you can manage patients in your office, but are also useful and recognizable by insurers, by Medicare, by Medicaid, and by others in the policy system that show this direction is what we need to do to create a sustainable health care system for the long run in our country?

We are not just exercise folks. We are not just gyms. But we do bring highly trained, mostly masters prepared exercise physiologists, registered kinesiologists, nurses, dieticians, and other professionals to create a team. So if you as a physician have a patient that you're trying to get to change their lifestyle, we hope to get from you the motivation for the patient, the "kick in the pants," if you will, in writing and a prescription that says, "you need to do some exercise. I want you to go look up Bob over here and he is going to help you with a plan." Then we will measure and monitor baseline biometrics and all the clinical endpoints that are important, physiometric measures that allow us to progress people along an exercise continuum, and exercise data that provides feedback and motivation to the person. We will develop an individualized program based on lifestyle, based on your current health status, based on the health risks, and any limitations we might receive from the referring physician.

We will provide that individual with the support, training, coaching, and nutritional assistance that they need to create habituation of positive lifestyle habits. That, for me, is the biggest key for all of this. We need to create good lifestyle habits as habituation and that takes 6 or 8 weeks to make those things happen. So we are seeing 8- to 12-week programs pop up across the country that are designed with this effort in mind to create those lifestyle changes and habituate them so that they are more sustainable in the long run. In short, that is what we in the medical fitness industry do. That is also why I am excited about our partnership with lifestyle medicine physicians. I believe our visions are closely aligned.

My goal by being here is to introduce what we do to you and look for ways to partner with you in order to gather the outcomes data to prove the value of the team approach. Anecdotally, we are seeing reduced readmissions to hospitals, reduced cost of care for patients overall, and a healthier patient base.

A cardiologist friend of mine has built a wellness center attached to his practice. He has a big 4-ft. tall clear trash can that sits in his lobby and, as his patients are able to get off of medicines, he has them drop their empty pill containers in the trash can. What better advertisement can you have for your patients when they come into your lobby to see that you come here to get well-to get rid of the chemicals that you are putting into your body?

What we are doing is real. It really works. There are good outcomes data available though it is mostly anecdotal at this time. The Medical Fitness Association is beginning 3 national studies looking at hypertension, diabetes, and obesity to look at the effectiveness of exercise and nutrition as a primary treatment in patients with these conditions. It is exciting to see what you are doing here at ACLM, not only demonstrating the effectiveness of lifestyle medicine but also gathering the hard data. With more good data, it will be easier to get people's attention. More physicians will begin to understand the value through the data leading to more supporters of lifestyle medicine.

Dr Rippe: I want to offer a couple of comments about what Bob said. I believe when it comes to lifestyle change, particularly in the area of exercise, we face what I would call the "Geico dilemma." The dilemma is this. You see the Geico commercials over and over. A person says "you can save 15\% on your insurance with Geico" and the other person says "everybody knows that!" Well guess what? When it comes to physical activity and health benefits, "everybody knows that!"

In USA Today they periodically publish snapshots and I noticed one where more than $90 \%$ of people agreed with the premise that physical activity is good for your health. What we should focus on is not whether or not people believe exercise is good for their health. In the words of the Geico commercial "everyone knows that!" What we need to focus on is the difference between what people know and what they are doing. That's the gap and that is where we should focus most of our attention.

I should also mention that one of the reasons why I have the pleasure of moderating this panel discussion is because I am the Editor in Chief of the American Journal of Lifestyle Medicine $(A J L M)$, which is the official journal of the American College of Lifestyle Medicine. The September/October 2015 issue was devoted entirely to this conference from the previous year. Members of ACLM know that because they receive $A J L M$ as part of their membership in ACLM. If you do not belong to ACLM currently, you can receive the September/October 2015 issue of $A J L M$ for next 60 days because we have allowed it to be published on the ACLM website. Please consider joining ACLM and you will get AJLM free every 2 months as part of your membership.

Dr Rippe: Here is the second question for all of the panelists to offer opinions about: Describe what population health management means to you and what role does lifestyle medicines play in population health management (PHM)?

Dr Parkinson: One of the things we love to do in health care is come up with the buzz words du jour. The buzz word du jour is "population health management." Those of you who are trained in public health, probably thought you were doing population health management all along! For those of us who went into family medicine to take care of family members in a community, we thought we were also doing population health management. Beware. When you can take any day of the week in Washington and find a conference named "Population Health Management" you know we may be in trouble. 
Semantics aside, population health management IS here and likely here to stay as it becomes more mainstream in health care. For example, at UPMC we have 62000 employees and use a population health management model to improve health and reduce excessive health care and productivity costs. Using this model, we partnered with the Advisory Board to establish Evolent Health, a now 1000-employee company essentially born of the ACA. Evolent Health is assisting hospitals and health systems nationwide to implement population health models to advance the "Triple Aim": improve health, improve patient experience, and reduce the per capita cost of care. And, over time, to take economic risk in doing so.

Digging deeper, what is a "population" in the context of population health management? First of all, I often say to doctors and others "there is no single 'population,' there are many tribes within a population." Populations are made up of socially, clinically, and anthropologically distinct units namely, people. And families. When they share common experiences, perspectives, values they form "tribes" with certain common challenges, attitudes, and behaviors. Individuals, employees, parents, colleagues don't declare themselves members of a "disease" tribe: as diabetics, asthmatics, or cancer patients. They are people first who could learn from and be supported by other people like themselves-a tribe if you will. But we don't take that perspective or capture that information in current population health models. True, we can better integrate and stratify all types of sophisticated metrics and we can even use advanced statistical techniques to perform "predictive modeling." But if we don't understand or embrace the tribes within the population we're trying to improve, we'll make little progress in preventing, treating, or reversing disease either in the short run or longer term.

Meta-analyses of successful behavior change show that three pillars are required: basic knowledge, new skills, and ongoing support.

The same is true for providers in health care. Seven years ago, the American College of Preventive Medicine, led by Drs Liana Lianov and Mark Johnson, convened a process to define lifestyle medicine competencies for physicians. We pulled together internists, pediatricians, family doctors, preventive medicine, lifestyle medicine, and sports medicine physicians and asked "What did we all miss in undergraduate medical education, residency training, and even fellowships? What knowledge, skills and support are necessary for physicians and medical practices generally to address and successful reverse the root causes of most all chronic diseases?" Knowledge about the relationship of lifestyle to disease causation is critically important and too often given short shrift. We dumb down the evidence of healthy eating, physical activity, sleep, healthy responses to stress to major diseases for both patients AND providers. Do we take the time to share with our fellow physicians and staff-no less the patients themselves-how most common chronic diseases are inflammatory in nature and that that inflammation is a direct result of what we eat, how we move, and how we think? If you give short shrift to the basic, emerging, and exciting science that underlies disease causality well, you're not going to get true population health improvement through "better medical care." Even "team-based care" may become a buzz word du jour unless we're careful. Team-based care for team-based care's sake is not an inherent good in and of itself. But teams can be particularly effective if it doesn't get people out of the hospital faster, measurably improve health, and reduce the total cost of care, I am not interested in paying for it. Neither are employers. Do the professionals on the health care team embrace the competencies of lifestyle medicine and do they assist patients to acquire the skills improve their health behaviors? Let's commit to better education about the root cause of disease, specific skill building of patient and providers using lifestyle medicine competencies and create sustainable environmental and other support systems to insure lasting impact. Then "teamness" will definitely contribute to improving population health. Knowledge-check. Competenciescheck. Number 3, a support system for both patients and providers. In any population health management plan, we will need to create a supportive and reinforcing environment around that person, family, employer, practice or health care professional. We all need someone to pat us on the back or kick us in the butt and say "you know what, you let me down today." Or better yet, "Great progress . . . keep it up!" Our patients and families need have to have new skills and support, which means somehow we or in partnership with others, are going to have to go into their homes, schools, and worksites and get the junk food out of the refrigerator and the cupboard. Who is going to do it? How can we do it? Without ongoing environmental support for our patients or ourselves as providers the "behavior change" we're seeking, that which we have initiated with knowledge and skills won't "stick" or be sustained in the long run.

What are we doing to advance lifestyle medicine at the very large, UPMC Health System that sponsors the third largest GME (Graduate Medical Education) program in the country? How are we making progress on lifestyle medicine using a population health framework at an established academically oriented integrated health care delivery and finance system? We successfully piloted the plant-based, whole food Complete Health Improvement Program (CHIP). We enlisted lifestyleminded physician leaders in cardiology, endocrinology, and bariatrics and enrolled some of their sickest patients in the pilot along with health plan members with multiple health risk factors identified in our wellness program. And we saw dramatic changes in the enrollee's biometrics and in our physicians' sense of professional satisfaction. Unfortunately however, we didn't have as many high-cost, complex patients on multiple medications to a cost reduction in lower 
prescription drug use in 6 months. But I know it's possible. And I want to reduce that cost. I want to reduce expensive recurrent medical events which then follow the reduction in prescription drug use as the cause of the underlying disease decreases or even disappears.

So lifestyle medicine must roll up its sleeves, get "dirty," and embrace population health management. And we have to get off the bully pulpit and familiar cry of "you should pay me more money." We've got to go find the people with 7 or more different doctors, 9 or more different medications, and get them engaged in lifestyle medicine, which becomes the foundation of their care management plan.

At UPMC, we are also using Six Sigma quality improvement models to "medicalize" behavior change by integrating doctors' orders for lifestyle medicine into our EPIC EMR (electronic medical record). Our physicians can now write prescriptions for healthy eating, physical activity, weight loss, and stress management from their desktops. We are moving toward a permanent presence of the CHIP program in the UPMC cardiology clinic. Our early results were so impressive that the Chairman of Cardiology said "I am going to start up the first dedicated lifestyle medicine clinic at UPMC." We are going to have a UPMC-wide lifestyle medicine conversation that every hospital CEO, every Chair of every division, all the clinical leaders to explore what the movement means for organized medicine in leading medical center. This is transformational. Lifestyle medicine is a movement-backed by ever-growing science. And we better get in front of it.

Population health can be lifestyle medicine's friend-but only if we roll up our sleeves and get involved. Amy's point is true. The vast majority of ACOs have not saved a nickel. Less than $10 \%$ of them did. Medical homes? THIS is my medical home (holds up cell phone). Medical homes similarly haven't saved any money. Yes, ACOs and medical homes have been shown to improve quality measures-at least those we currently measure. But let's get back to
Dexter's plea. He needs to save money now creating high-performing employees to compete globally. He's expecting health care to apply techniques like Six Sigma-which Cummins uses every day to produce highest quality, defect-free diesel engines. Employers can't wait for a 5-year lead time to show economic benefit. We need to crank it up and people in this room can contribute to the challenge of poor quality and excessive costs in a very constructive way!

Dr. Mechley: Ok population health. I think it is one of the most misused terms out there. Let's break this down to 3 things. First, define your population. You can do population health in a school system, in a community, in my practice, in a large hospital system. Second, understand the health outcomes and disparities of that population. You need to have data on that population to do so. Third, population health management becomes "Can you do something about it? Can you change certain defined health outcomes or decrease disparities?" Typically, this is done with a defined initiative or goal. That is population health management.

What it is not, in my opinion: Population health management has been misused as a cost strategy, as a way of bundling, as a strategy for cost shifting, which makes me a little crazy because that is short term, quarter to quarter concept and that is not effective for long-term health outcome change. So what I see is the term population health management being used to shift risk, from one entity to another.

To really change health outcomes, lifestyle medicine is the most effective and sustainable way. I do believe in ITLC (intensive therapeutic lifestyle change) programs. These programs change behaviors and therefore change communities, long term. In such a way, you are changing the drivers, getting to the root cause of health issues and then typically lifting all ships. You therefore are accomplishing population health management.

Also to do this well, we need good data. One of the things I believe is critical is the intraoperability of HIEs (health information exchanges), a clearinghouse of health care data. This is where the state and federal government can have significant impact; because with data we can all make a difference.

Ultimately, a delivery model infusing lifestyle medicine with primary care will be the most effective for large populations. At The Christ Hospital, we have been able to illustrate the power of primary care with some current data analysis of the Ohio region. In 16 practices that I have helped oversee in stages of transformation, we analyzed total cost of care data in 5 chronic disease areas, namely-CHF (congestive heart failure), COPD (chronic obstructive pulmonary disease), diabetes,

hypertension, and hyperlipidemia.

Because of the passion and interest of Dr Jeremy Bruce, we noted that patients that regularly saw their primary care doctor had a lower total cost of care as well as a lower specialist spend, even though they had the same disease burden as those with a higher cost of care. The total cost of care was inversely proportional to the percentage of money spent on primary care. So if a patient who has 7 or more visits with a specialist visits a primary care doctor once in that year, the total cost of care is decreased by $69 \%$. I looked at these data and said "there is no way, that's got to be wrong." So he did it on a larger cohort. We have challenged Medicare to do the same because we have shown it across 44 physicians an average decrease of $84 \%$ reduction in total cost of care across all 5 conditions, if primary care is involved in the treatment of that patient, minimum 1 visit. That's the power of primary care!

So when I look at systems and consider what we need to do, I think we need to really look at empowering primary care to be the engine of this transformation of a population. We need to make sure that we are resourcing primary care physicians appropriately, infusing lifestyle medicine. That is interoperability with health care data in a defined population and we all understand and share the vision of what those changes are going to be! 
Mr Porter: Quite simply, population health is redefining responsibility for the health and the cost of care for a population to those who have the greatest degree of opportunity to influence and improve that result and that resides with the individual. Redefining the relationship with your care team, with the community and resources that surround them is important.

You really need to define and understand the population, stratify them, and be able to make sure that you have an individualized plan of care that reflects the reality of what that person deals with. You need to redefine, again, the traditional health care system along of the lines of what Amy was describing to redefine it as a relationship that actively engages that person, but you also have to be able to deposit population medicine in the context of the larger community and environment to make sure the person has the ability to access the resources and be able to make those good choices that right now are not easy for many people to make. It really would help patients to optimize their well-being and health.

So population health, lifestyle medicine, is an element that I like to use to define for an individual that lifestyle formula that will most enable him or her to be able to achieve optimal health status with the least amount of cost. To get rid of all the process failures that people who have avoidable illnesses or encounters experience because they are living an inherently heathier life. But lifestyle medicine sits within a broader context. We have become an instrument that activates a change in a larger system that enables that individuals to be able to be more compliant and supports them in the sustained behavior change.

Dr Shurney: For me, population health is a very simple concept. It is the health status of a population; however, you decide to define that population. Is it getting better or worse? If you are being successful in population health then the health status of that group improves in time. If you're not making progress, it goes the other way. We live in a society where it seems it's going the wrong way. For the most part, we are not being very successful at population health.

What does population health look like? It looks like the Blue Zones. It looks like The Way to Wellsville Projects and what's being developed in those communities. It looks like Rockford, Illinois, where the CHIP program has been going gang busters. Those are prime examples of what it looks like. Notice I didn't say it looks like having more medical technology or facilities. It looks like when you have conversations about research that was just published like past week on the link between beating cancer by eating less meat and your oncologist is actually talking about that instead of ignoring it. Shouldn't this be an integral part of the conversation when oncologists are talking to their cancer patients about survivorship? It looks like not having to fret about your pharmaceutical (drug) costs increasing an estimated 30\% next year due to the introduction of something called PCSK9s for high cholesterol because you have a lifestyle medicine program that reduces cholesterol in a matter of weeks and is far less expensive. Why pay $\$ 12000$, $\$ 14000$ a year for a drug that you really don't need to spend money on? That is what it looks like and it is through a real lifestyle medicine approach to care that we are going to get where we need to go. It is the only way.

That is population health and the physician plays a vital role. Although the physician may not be the one that is performing every coaching encounter, the physician does needs to lead the charge. The patient and physician both need to be knowledgeable in order to do this well and that is where shared decision making comes in. Also the physician needs to model those behaviors and adopt those behaviors in his or her own life because otherwise they will not be seen as genuine. If it's so good for me to do, why aren't you doing it? How do you motivate someone to do something that you can't motivate yourself to do? It is very difficult. So that is another example of what it looks like.
At Cummins, we have something we call $\mathrm{MC}^{2}$ that we frame our population health efforts around with the goal of sustainable behavior change. "M" stands for Motivation. You have to motivate people in order to get them to change. There has to be something in it for them. We have to answer the question, "What's in it for me?" What I have found is that it has to be more than a monetary incentive. It has to be more than a competitive challenge here and there. It has to be something that inspires and makes the person feel better in order for them to stay engaged.

One of the things that I am particularly happy with is that in one of our lifestyle programs we focus on sleep. Sleep is a powerful motivator because you feel better the very next day. When you teach someone how to get a better night's sleep, even when you can't extend the hours of sleep, they feel better immediately. That's a powerful motivation. So what's in it for me, and how does it make me feel are questions and the answer is different for every individual patient and likely will change over time. So we must be astute and build programs that are not a one-sizefits-all approach.

Once we motivate a person, then we have to make sure they have the competencies and skills to be successful as they act on their motivation. The first "C" stands for Competencies. We have to make sure they have the right competencies. They must have the skills, not just the knowledge. They need the skills to incorporate change into their daily lives. If they don't want to run for 20 minutes or 60 minutes or whatever we are telling them to do, maybe we can get them to just stand up more. That is a lot easier to do. They are still moving. They are still increasing their movement. We need to teach them how to do that. It is more than just the acknowledgment. It is how they incorporate that into their lives. There is something called N.E.A.T, which is Non-Exercise Activity Thermogenesis. Many of you are familiar with this concept. Teaching people how to build an extra 500 calories of energy expenditure into their lives on a daily 
basis in seamless ways. That represents over 50 lbs. a year in weight loss, if done right. That's powerful!

The second "C" is Culture. We need to build a healthy lifestyle culture around the individual. Once you have motivated them to change, and they have acquired the skills (competencies) of how and what to do, then the cultural environment must be such to support the desired change. Otherwise, it will be very difficult for the individual to maintain that change. So $\mathrm{MC}^{2}$ is how we frame and develop all of our strategies directed to individual behavior change and what is necessary to sustain it and move it forward. And, that is what has occurred in those populations and in those cities I named here a few minutes ago.

Mr Boone: Population health is pretty much defined in the eye of the beholder. It really depends on your perspective. If you are an employer, it is your employees. If you're a health system, it is the entire population that you serve. If you are a community, it is your entire community. Notice the difference among these. If you are a physician, it is probably your practice panel. So when you take a look at population health in these different ways, what it boils down to is managing the risk of a defined population. Risk is going to affect health. Expenditures for managing that risk is going to be critically important. It is also going to come down to managing recidivism. Helping populations stay healthier through lifestyle interventions will help to keep people out of the hospital in the first place and also affect readmissions.

So really what we are talking about with population health management is how we manage the risk of the patients that we serve. In order to do that, I would like to challenge each of you with a little quiz. How many of you have analyzed the risks of the patients in your own practice? How many of you know what percentage of your patients have made specialty visits within the past year? Do you know what the average cost of care for each of your patients is? If you have not done the work of analyzing your own practice, how can you hope to manage the health risks within that practice? How can you prioritize where your practice needs to focus to have the greatest impact on your panel? How do you know what resources to deploy? How do you know who to partner with to best affect your patients? Should you bring on a health educator in your own practice? Should you contract for these services, or refer? How do I determine whether direct contracting is a good solution for my practice? Understanding the dynamics of the practice and the utilization by your patients as well as evaluating their risks will allow you to answer many of these questions.

Over the past couple of years we have partnered with a group called the Association for Corporate Health Risk Managers. This has been very eye opening to me coming from a hospital world to understand the different kind of approaches that the business community is taking toward managing their health care dollars and the health risks of their employees. We are already seeing in certain parts of the country direct contracting efforts with health systems and doctors' practices, the building of very narrow networks, and other efforts to manage risks. If we in health care don't step up to the plate and begin to actively manage that risk, it is going to be managed for us.

My admonition to each of you is to analyze your practice. Understand your patients. Understand where the opportunities lie and begin putting together a strategic plan with strategic partners that address all the issues the panel has been discussing. If we don't do this, we might very well find ourselves left out when those direct contracting approaches and other innovations happen.

Once you understand the patterns of your practice, then there is a basis for creating an active plan for your practice. By organizing your patients into disease cohorts, you may consider scheduling a group of your diabetic patients scheduled in a 4-hour block that would allow you the opportunity to provide group education, intervention, and management utilizing some of the new CPT (Current Procedural Terminology) codes while improving the consistency, efficiency, and effectiveness of the services provided to the patients.

To me, population health means we are identifying, coordinating, and providing services to segmented populations utilizing resources in the community that will best help provide care for your patients. Under the new payment methodologies, primary care physicians are being held responsible for the health of their panels. How can this be done given that in general, in a busy family care practice, each patient may only be seen twice a year for 10 minutes. What resources are available to go to individuals' homes and get out all junk food in the kitchen? What resources will be available to assist patients in creating these new lifestyle habits? How are we going to find those resources to do that in a costeffective and meaningful way? That's the $\$ 64000$ question to be sure. But there are available community-based resources.

We are beginning to hear the term value-based care in addition to population health. Value-based care is broken up into community care, institutional care, and into physician practice care. The question, then, is how do we mix and match the best components of each in order to manage that overall risk and create value? Here is where we still have some work to do in generating the programs and evidence based outcomes that demonstrate the clinical effectiveness and cost efficiency of community-based care. Then we will be providing population health management one person at a time.

Dr Rippe: I am now going to change our format a little bit and ask each of the panelists to very briefly answer a series of questions. Question \#1:

Please tell us of a novel idea or program that you have participated with or are familiar with that you feel exemplifies the concept of lifestyle medicine as a key component in the bealth care transformation or PHM. 
Dr Parkinson: Prescription for Wellness. Come tomorrow afternoon and hear about it.

Dr Mechley: I would say direct primary care. Think about the value proposition for your patients who you serve and the value proposition for your providers. When you have something that has a value proposition for both, then we really have something!

Mr Porter: The Ornish Program certainly is an innovative program for intensive cardiac rehab treatment of cardiac disease but I am going to use an unusual approach. The most innovative program that I have been familiar with is the longitudinal study of Marie Porter's care plan. My 93-year old mother just passed away in August and 14 years of piecing together the elements required to optimize her lifestyle, not letting the medical system alone define her life. It involved taking responsibility to interact with her physician and together craft for her a plan for how she could optimize the quality of her life, her social relationships, her diet, her transportation, her movement, and so on. I learned a lot over that 10-year period and we are woefully deficient in creating the best life for people who have much to offer.

Dr Shurney: I would say taking the concept of nutritionfacts.org and expanding it into lifestylefacts.org so it is more than just nutrition. It is all of those other aspects of lifestyle to make it a comprehensive tool and resource. I am delighted that the American College of Lifestyle Medicine foundation is actually doing this important work.

Dr Rippe: Do they own that URL?

Dr Shurney: Yes they do.

Dr Rippe: Everyone should contribute to that!

Mr Boone: We see the development of 8- to 12-week programs for zero or very little cost of entry that do habituation lifestyle coaching, exercise, and lifestyle intervention and prevention.
Dr Rippe: In medicine, we tend to be splitters rather than lumpers. So we talk about diabetes, obesity, hypertension, and cardiovascular disease. That to me is essentially manifestations of one underlying disease. A lot of people are calling that "cardiodiabesity" to indicate that it is a continuum of one disease. These individual diseases or risk factors are simply different manifestations of the same underlying problem. There are different theories of the underlying problem but they all interact very, very strongly.

Second, I want to mention that there was a wonderful article a few years ago in the New England Journal of Medicine by Michael Porter, a Harvard Business School professor, who said the major problem in American medicine is that we have "lost the value proposition." We no longer deliver value. Value is better outcomes without increased costs and the only way we can do that is to adopt the practices that we are talking about here.

The third issue I want to raise is one that I ended my editorial with after last year's expert panel discussion when I wrote in the $A J L M$ using a quote from Margaret Mead who said "Never doubt that a small group of thoughtful, committed citizens can change the world; indeed, it's the only thing that ever has., ${ }^{\prime 4}$ I believe it is critical at a conference like this to recognize and support each other as that small group of thoughtful, committed citizens who truly can change the world. I am proud of all of you who made the effort to attend this conference and thankful to the people who organized it because these are the kind of events that rekindle enthusiasm and belief for participants that you are doing the right thing for your patients, and in the final analysis, for our country! I want to conclude with a quote that came from a tee shirt that my wife and I saw at one of the swim meets that one of my children participated in. The quote said "Without a dream, there is no reason to work. Without work, there is no reason to dream." I hope and believe that by participating in this conference and meeting the other people who are as passionate and committed as you are, you will be more invigorated to work every day to fight the fight. We all need to keep fighting for what we know will ultimately be required if we are going to improve the health of our country by dealing with the root causes of what are making us sick.

Every 2 years, the Department of Health and Human Services asks people about their health. Reliably, $80 \%$ to $90 \%$ of people say that their health is either "good" or "very good." It is amazing that despite the facts that fewer people are exercising, over two-thirds of the adult population is overweight or obese and most people are eating poorly, reliably $80 \%$ to $90 \%$ say that there health is "good" or "very good." How can this disparity exist? I believe that it is because most people are framing health in the wrong way. They said "I don't have a chronic disease, therefore, my health is good." What they are not saying is "I am taking those steps in my daily life that will lower my risk of chronic disease and make me feel and perform better and be happier."

I have learned a long time ago as a physician that nobody likes to be sick. However, many people do not know that they are sick. They don't know that they are doing things that 10 or 15 years from now could result in a leg amputation or blindness or quadruple bypass surgery because at the current moment they have framed their health as "I do not have some dreaded disease therefore my health is good or very good."

We need to fundamentally transform that current form of thinking into people understanding that good health is God-given and that we should all be trying to preserve it. That is our job. Members of the audience, I urge you as that small group of committed people, who are going to change the world to make sure that people understand the distinction between "good" health and "optimal" health and use that in a motivational way. This is something I have devoted the past 20 years of my life to and God willing I will have another 20 years to be able to keep coming back and be inspired, 
myself, by seeing all of you and how much passion, commitment, and hard work you bring to the very important area of lifestyle medicine. Thank you all for coming and participating in this opening session of this year's ACLM National Conference.AJLM

\section{References}

1. Rippe J. Lifestyle Medicine. 2nd ed. Boca Raton, FL: CRC Press; 2013.

2. Irwin RS, Rippe JM. Irwin and Rippe's Intensive Care Medicine. 7th ed. Philadelphia, PA: Lippincott Williams \& Wilkins; 2011.
3. Porter M. A strategy for health care reform-toward a value-based system. $N$ Engl J Med. 2009;361:109-112.

4. Mead M. Quotation \#33522 from Laura Moncur's motivational quotations. http://www.quotationspage.com/ quote/33522.html. Accessed November 20, 2015. 